\title{
Tendencia al reemplazo de Animales de Experimentación
}

\section{Trend to replacement of Experimental Animals}

\author{
Martín Nicolás Rivero artinnicolasrivero@gmail.com
}

https://orcid.org/0000-0001-5863-7061

Laboratorio de Métodos Alternativos de la Plataforma Tecnológica EBAL; Instituto de

Farmacología; Facultad de Medicina; Universidad de Buenos Aires (Argentina)

\author{
Mariela Lenze: marielalenze@gmail.com \\ https://orcid.org/0000-0002-7696-7950
}

Laboratorio de Métodos Alternativos de la Plataforma Tecnológica EBAL; Instituto de

Farmacología; Facultad de Medicina; Universidad de Buenos Aires (Argentina)

\section{Mercedes Izaguirre: merce.izaguirre@hotmail.com}

https://orcid.org/0000-0003-2275-0614

Laboratorio de Métodos Alternativos de la Plataforma Tecnológica EBAL; Instituto de Farmacología; Facultad de Medicina; Universidad de Buenos Ares/ Consejo Nacional de Investigaciones Científicas y Técnicas 
Martín Nicolás Rivero, Mariela Lenze, Mercedes Izaguirre, Silvia Wikinski y María Laura Gutiérrez Tendencia al reemplazo de Animales de Experimentación

\author{
Silvia Wikinski: silviawikinski@gmail.com \\ https://orcid.org/0000-0001-6501-2916 \\ Laboratorio de Métodos Alternativos de la Plataforma Tecnológica EBAL; Instituto de \\ Farmacología; Facultad de Medicina; Universidad de Buenos Aires/ Consejo Nacional de \\ Investigaciones Científicas y Técnicas
}

María Laura Gutiérrez: mlgutierrez.ebal@gmail.com

https://orcid.org/0000-0003-4000-7816

Laboratorio de Métodos Alternativos de la Plataforma Tecnológica EBAL; Instituto de Farmacología; Facultad de Medicina; Universidad de Buenos Aires/ Consejo Nacional de Investigaciones Científicas y Técnicas (Argentina)

\title{
Resumen
}

Para la aprobación, registro y comercialización de medicamentos, agroquímicos, productos de uso doméstico y sustancias cosméticas es necesario estudiar e informar al organismo regulador la potencial toxicidad. Por motivos éticos y económicos, en los últimos años se ha disminuido la utilización de animales de experimentación y se ha fomentado el desarrollo de metodologías alternativas. En el proceso de migración, la OCDE validó procedimientos para evaluar irritación y corrosión ocular y dérmica, mutagenicidad, fototoxicidad, sensibilización y absorción dérmica. Hoy en día es un área en constante investigación y desarrollo ya que aún no se ha logrado reemplazar completamente la calidad de los datos obtenidos en animales. Si bien la tendencia mundial al reemplazo de animales es clara, en Argentina aún no hemos dado el primer paso: no contamos con una ley que regule el uso de animales de experimentación. Las agencias reguladoras (SENASA y ANMAT) continúan solicitando ensayos con animales, pero han manifestado su interés en aceptar evidencia toxicológica obtenida por métodos alternativos. Desde el Laboratorio de Métodos Alternativos al uso de Animales de Experimentación que forma parte de la Plataforma Tecnológica EBAL nos hemos propuesto trabajar en el desarrollo, ejecución, perfeccionamiento y transferencia de metodología alternativa.

Palabras clave: toxicología, métodos alternativos, principio de las 3Rs 
Martín Nicolás Rivero, Mariela Lenze, Mercedes Izaguirre, Silvia Wikinski y María Laura Gutiérrez Tendencia al reemplazo de Animales de Experimentación

\section{Abstract}

For the approval, registration and commercialization of drugs, agrochemicals, household products and cosmetics substances is necessary to study and inform the regulatory organism about the potential toxicity. Due to ethical and economic reasons, the use of experimental animals has decreased in the last years and the development of alternative methods has been improved. During the process of replacement, the OECD validated procedures to test dermal and ocular irritation and corrosion, mutagenicity, phototoxicity, dermal sensitization and absorption. Nowadays this area is in constant investigation and development as it has not been already possible to completely replace data quality obtained in animals. Even though the global trend to replace animals is clear, in Argentina the first step has not been taken yet as there is not a law that restraint the use of experimental animals. Although procedures with animals are still requested by the regulatory agencies (SENASA and ANMAT), they have shown interest in accepting toxicological evidence obtained by alternative methods. In our Laboratory of Alternative Methods to the use of Experimental Animals which belongs to the EBAL Technological Platform we have proposed to work in development, execution, improvement and transference of alternative methods.

Keywords: toxicology, alternative methods, principle of the 3Rs

¿Cómo surge la implementación del uso de animales para evaluar la toxicidad de sustancias de uso humano?

En 1933 la ceguera producida en una mujer por la aplicación de una máscara de pestañas (Figura 1a), llevó a que la Administración de Alimentos y Medicamentos (FDA, por sus siglas en inglés) de Estados Unidos exigiera ensayos de seguridad para la aprobación de productos cosméticos. En 1944 el toxicólogo John Draize (Figura 1b) de la FDA diseñó una prueba de toxicidad aguda en conejos, conocida como "test de Draize". Existen dos variantes de la misma: la prueba de irritación cutánea aguda y la prueba de irritación ocular aguda (Mindel \& Wilhelmus, 2001). La prueba de irritación ocular aguda consiste en la aplicación del producto en conejos albinos durante 21 días a lo largo de los cuales se observa si ocurrieron lesiones en la córnea, iris y conjuntiva. En el caso de la irritación dérmica se aplica el producto en la piel de conejos previamente rasurados. En ambos se da una valoración numérica según el grado de lesión ocasionada. 
Martín Nicolás Rivero, Mariela Lenze, Mercedes Izaguirre, Silvia Wikinski y María Laura Gutiérrez Tendencia al reemplazo de Animales de Experimentación

Entre las innumerables sustancias que potencialmente podrían generar respuesta tóxica se encuentran los productos farmacéuticos, cosméticos y artículos de tocador, productos utilizados en la limpieza del hogar, la industria y la agricultura. La prevención del daño causado por agentes potencialmente tóxicos es un objetivo de Salud Pública. En este sentido tanto los organismos reguladores como los investigadores estamos involucrados en la predicción de seguridad de los compuestos que luego llegarán a manos de los usuarios o de los pacientes.

a

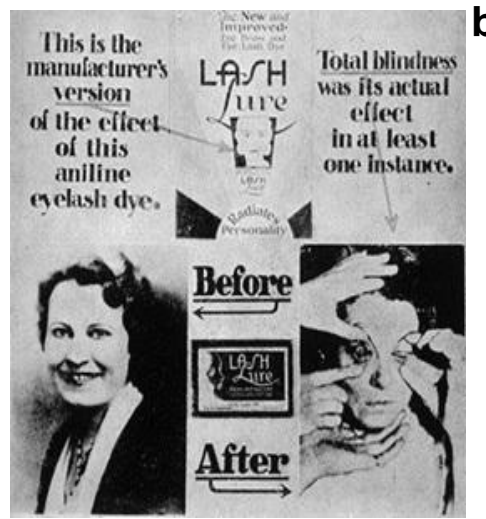

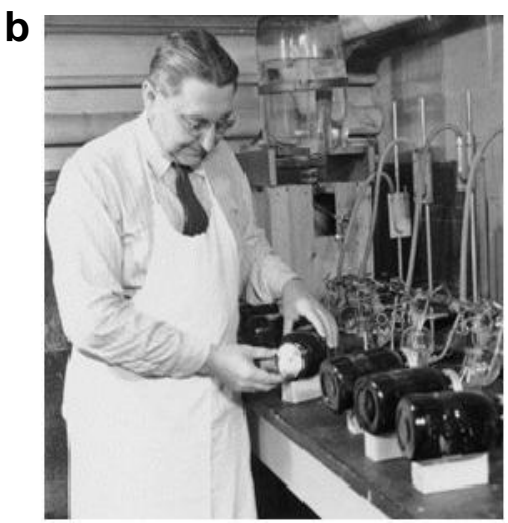

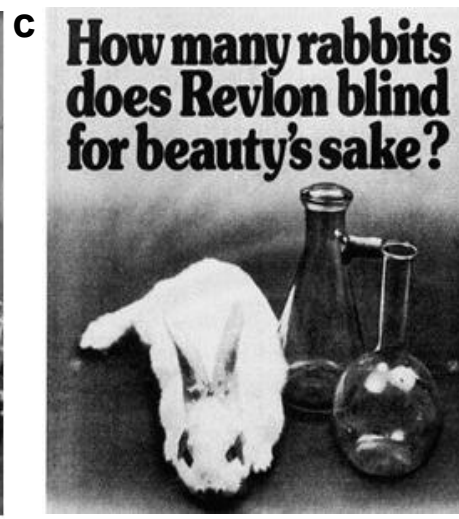

Figura 1: a) Recorte periodístico de la época de un caso de ceguera producido por un cosmético; b) John

Draize, farmacólogo de la FDA, quien desarrolló métodos para la evaluación de la toxicidad ocular y dermal de drogas y cosméticos y c) Crítica periodística sobre el test de Draize publicada en el New York Times en 1980. Las imágenes fueron tomadas de Mindel, J. \& Wilhelmus, K. R. (2001). The Draize Eye

Test. Therapeutic Reviews, 45(6),493-515.

Animales en desuso: un cambio de paradigma

El test de Draize ha tenido diferentes adaptaciones y mejoras a lo largo de los años, incluso a nivel de la OCDE (Organización para la Cooperación y el Desarrollo Económico). Sin embargo, el procedimiento ha sido ampliamente criticado (Figura 1c) no solo por sus aspectos éticos en relación con el uso de animales, sino también por tratarse de metodologías subjetivas en los que la puntuación otorgada depende de la experiencia del observador, además de las diferencias existentes entre el ojo y la piel del conejo con respecto al humano.

En las últimas décadas el debate en torno al uso de animales de experimentación y el derecho animal ha crecido considerablemente. Los modelos animales utilizados en ensayos de seguridad de productos han comenzado a ser cada vez más discutidos. Ya en 1959 William Russell y Rex Burch publicaron "Los principios de las técnicas experimentales humanitarias", conocido como "Principio de las 3Rs". Las erres hacen referencia a Reemplazo, que es la 
sustitución de animales por material no vivo o insensible siempre que sea posible; Reducción, que implica utilizar la mínima cantidad de animales con la que se puedan obtener resultados confiables (lo que se puede estimar por medio de herramientas estadísticas) y Refinamiento, que se refiere a evitar procedimientos que lleven al sufrimiento del animal, minimizar el dolor y el estrés, utilizar anestesia y analgesia, asegurar el acceso a agua y comida, etc. (Balls, 2015; Russell, 1995). Si bien el concepto de las 3Rs no fue considerado con la seriedad pertinente en sus inicios, en las últimas décadas ha tomado fuerza al punto que hoy es la base del correcto trabajo con animales de experimentación. La industria, la ciencia y la política han demostrado compromiso con las $3 R$ s, lo que se ha convertido en un pacto entre quienes luchan porque los experimentos con animales desaparezcan y la industria que sigue necesitando de ellos. La aplicación del principio de las $3 R$ s indujo a nivel internacional un cambio en el enfoque experimental para la investigación de la toxicidad de sustancias con posible impacto en la salud humana o animal, proporcionando una estrategia para minimizar el uso y el sufrimiento de los animales de experimentación, sin comprometer la calidad del trabajo científico. Una prueba de este hecho es que los experimentos con animales disminuyeron en aproximadamente dos tercios desde su punto máximo, reportado a mediados de los años setenta (Hartung, 2017).

Muchas de las metodologías que se validaron con el fin de reducir y reemplazar son técnicas que utilizan cultivos de células de origen animal, las cuales se denominan metodologías in vitro. Dichas metodologías poseen puntos a favor y en contra. Entre las ventajas podemos afirmar que las condiciones experimentales están mejor controladas, que hay un alto nivel de estandarización y menos variabilidad, que las pruebas son más rápidas y en algunos casos hasta más económicas. Sin embargo abandonar los modelos animales tiene sus desventajas. Por ahora no hay métodos in vitro validados para evaluar los efectos sistémicos (en todos los órganos de un individuo en simultáneo) ni estudiar cómo se distribuye una molécula en el organismo, los denominados estudios de farmacocinética y de farmacodinamia. Otro punto importante a destacar es que los métodos alternativos requieren controles de calidad (Bal-Price \& Coecke, 2011) más exhaustivos y una alta capacitación del personal que los lleva a cabo. 
Martín Nicolás Rivero, Mariela Lenze, Mercedes Izaguirre, Silvia Wikinski y María Laura Gutiérrez Tendencia al reemplazo de Animales de Experimentación

Políticas de implementación de metodologías alternativas al uso de animales

En la Comunidad Económica Europea (CEE) el Parlamento dio lugar en 1986 a la Directiva 86/609/CEE: Protección de animales de experimentación y otros fines científicos. En la misma se menciona que "Un experimento con animales no debe llevarse a cabo si existe otro método científicamente satisfactorio que permita obtener los mismos resultados con una práctica posible y razonable" (European Parliament and of the Council, 1986). Esto dio lugar a que pocos años después, en 1991, se establezca en Europa el primer Centro de Validación de Métodos Alternativos (ECVAM, por sus siglas en inglés). Dos años más tarde, en 1993 surge la primera directiva con el objetivo de prohibir el uso de animales en ensayos de seguridad de productos cosméticos. La directiva puso un plazo de 5 años para el cumplimiento de la misma pero se fue posponiendo hasta que en 2004 pudo implementarse la primera etapa: la prohibición del testeo en animales de productos cosméticos terminados. Finalmente, la implementación completa se llevó a cabo en el año 2013 con la prohibición del testeo en animales de ingredientes y productos terminados dentro la CEE, y la prohibición de la comercialización de productos cosméticos que hayan sido testeados en animales tanto dentro como fuera de la CEE (Figura 2) (Council European Parliament, 2010).

\section{CONNEETINS THE DOTS FOR ANIMALS: HISTORY OF THE EU BAN ON ANIMAL TESTING FOR COSMETICS}

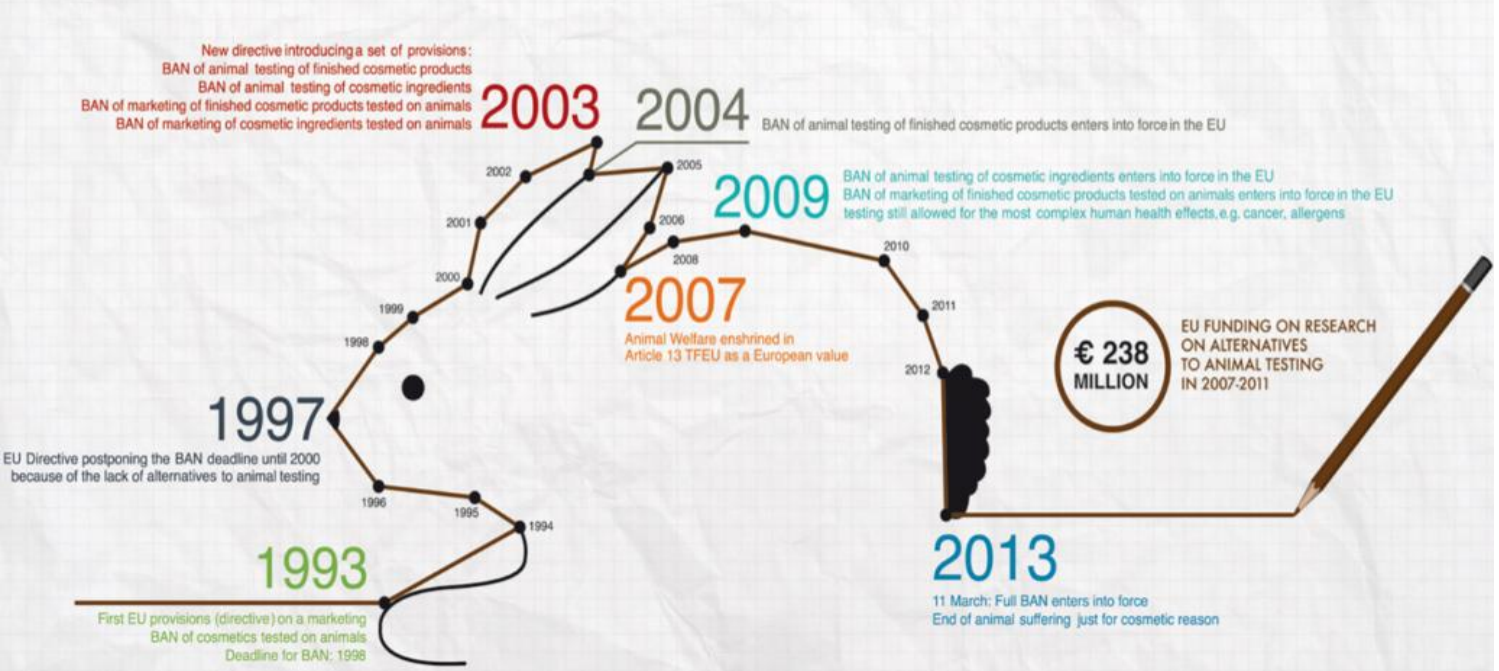

Figura 2: Línea temporal del proceso de reemplazo y posterior prohibición del uso de animales de experimentación para el testeo de cosméticos en la Comunidad Económica Europea. Tomada de "Ban on animal testing", publicada en https://ec.europa.eu 
Los métodos alternativos han ido ganando territorio en las diferentes agencias regulatorias, tanto que hoy se recomienda comenzar a realizar los ensayos de seguridad de cualquier sustancia, siempre que sea posible, por metodología in silico (utilizando modelos computacionales), in chemico (mediante reacciones químicas), in vitro (utilizando células en cultivo) y ex vivo (a partir de tejidos animales sacrificados para otro fin). En este sentido, la regulación europea para el Registro, Evaluación, Autorización y Restricción de Productos Químicos (REACH, por sus siglas en inglés) estipula que los experimentos con animales pueden llevarse a cabo sólo si los métodos alternativos no son aplicables o si los resultados no son adecuados para la clasificación y evaluación de riesgos (European Parliament, 2013).

En el largo camino recorrido desde la conformación del ECVAM a la actualidad, se han validado procedimientos alternativos al uso de animales para evaluar irritación y corrosión ocular, mutagenicidad, fototoxicidad, irritación, sensibilización, corrosión y absorción dérmica entre otros, todos ellos aplicables a productos fitosanitarios, cosméticos y farmacéuticos (Kandárová \& Letaáiová, 2011). Actualmente existe una amplia lista de métodos validados y aceptados por organismos internacionales, que potencialmente podrían comenzar a implementarse en la región.

\section{Situación a nivel regional}

Siguiendo los pasos de Europa, Brasil tomó de modelo la directiva 86/609/CEE que regula el uso de animales de experimentación y en el año 2008 se estableció en el país vecino la Ley 11.794, también conocida como Ley Arouca, que regula los procedimientos para el uso de animales en la investigación (LEI N ${ }^{\circ} 11.794$. Procedimentos para o uso científico de animais, 2008). Un año después en Uruguay surge la Ley No. 18.611 que establece las condiciones de uso de animales en experimentación, docencia e investigación científica, ley que también sigue el modelo de la ley europea (Ley 18.611 Utilización de animales en actividades de experimentación, docencia e investigación científica, 2009).

En el año 2013 se implementó en Europa la directiva que prohíbe la comercialización de cosméticos testeados en animales. Como consecuencia, el Estado de Brasil se planteó como objetivo invertir dinero en incorporar los métodos alternativos ya validados por la OCDE y así evitar la pérdida del mercado europeo, dado que este país es fuerte en la producción tanto de materia prima como de productos cosméticos terminados. Para ello en el año 2014 se establecieron dos fechas límites para la implementación de dichas metodologías (la RN 18/2014): la primera en septiembre de 2019 y la segunda en septiembre de 2022. El Congreso 
Martín Nicolás Rivero, Mariela Lenze, Mercedes Izaguirre, Silvia Wikinski y María Laura Gutiérrez Tendencia al reemplazo de Animales de Experimentación

de San Pablo, recorriendo su propio camino, sancionó en 2015 la Ley № 15.316, prohibiendo el testeo de cosméticos en animales dentro de ese estado brasileño.

En Argentina la realidad dista de la de nuestros compañeros del Mercosur. Frente a la tendencia mundial de reemplazo de animales de experimentación, que ha llevado al cambio de paradigma en la evaluación de seguridad de productos industriales, no se han tomado medidas de ningún tipo en nuestro país. En 2015 y en 2017, la senadora Magdalena Odarda presentó un proyecto de ley "Prohibición del uso de animales para el desarrollo, experimentación y pruebas de nuevos componentes de cosméticos y artículos de tocador y perfumes". La propuesta no prosperó en ninguna de las dos ocasiones ya que resulta impensado en un país que no regula lo más básico, como el uso de animales de experimentación, pasar directamente a la prohibición. Lo positivo es que dio lugar al debate y a la reflexión entre los interesados en el tema, ya sean tanto proteccionistas como científicos que trabajan en modelos animales. Por lo que se puso en evidencia la necesidad de dar el primer paso y contar con una ley que regule la experimentación animal y que contemple el reemplazo de animales siempre que exista una alternativa; es una etapa intermedia que ha garantizado el éxito en los países en lo que se han dejado de usar animales para el testeo de cosméticos. En este sentido, en 2017, el diputado Alejandro Echegaray junto a un grupo de científicos y otros legisladores dio forma a un proyecto de ley que planteaba regular la utilización de animales en laboratorios y bioterios. La propuesta, que contó con media sanción y luego perdió estado parlamentario, tomaba como modelo las leyes vigentes en Brasil y Uruguay, y se sostiene en el principio de las $3 R$ s utilizado en Europa. El proyecto de ley contemplaba la creación de la Comisión Nacional de Animales de Experimentación (CONADEA), la obligatoriedad de la evaluación de los procedimientos por Comités Institucionales para el Cuidado y Uso de Animales de Laboratorio (CICUAL), el registro de los bioterios ante la CONADEA para garantizar el cumplimiento de estándares mínimos que garantizan el bienestar animal y la aplicación de las 3Rs.

En mayo del presente año, la senadora Magdalena Odarda presentó un nuevo proyecto que promueve la prohibición del uso de métodos in vivo (en animales) para testeos que evalúan tolerancia dérmica y ocular de productos cosméticos y de perfumería. El mismo contempla la incorporación de metodologías validadas internacionalmente.

Por otra parte, en el transcurso de 2017 tuvieron lugar reuniones de un grupo de investigadores que, movilizados por intereses comunes de implementación de métodos alternativos al uso de animales en Argentina, conformaron RAMA, la Red Argentina de Métodos Alternativos. Con el fin de dar un puntapié inicial al trabajo realizado durante el año, se organizó con gran convocatoria en octubre de 2017 la primera Jornada de Métodos Alternativos al uso de Animales de Experimentación "Implementación de las 3Rs" organizada por RAMA y AACyTAL 
Martín Nicolás Rivero, Mariela Lenze, Mercedes Izaguirre, Silvia Wikinski y María Laura Gutiérrez Tendencia al reemplazo de Animales de Experimentación

(Asociación Argentina de Ciencia y Tecnología de Animales de Laboratorio) (1). Un año más tarde, Buenos Aires fue sede del COLAMA 2018 (Congreso Latinoamericano de Métodos Alternativos) ${ }^{2}$, que convocó a expertos en la materia tanto americanos como europeos. La organización de ambos eventos en nuestro país fue un estímulo para que tanto los organismos reguladores como la industria perciban que la llegada de los métodos alternativos al país es inminente.

Implementación de métodos alternativos en Argentina: un beneficio para la industria nacional

El uso de agroquímicos implica un posible riesgo para la salud tanto de los trabajadores que aplican los productos como para la población cercana a las superficies cultivadas. Por este motivo, en el proceso de registro ante SENASA es obligatorio presentar información que permita estimar su potencial toxicidad a través de ensayos en animales (Secretaría de Agricultura, Ganadería, 2012).

Para el registro de productos cosméticos y domisanitarios (productos de higiene domiciliaria) ante ANMAT también es necesaria la presentación de evidencia toxicológica. La aprobación de numerosos productos está condicionada a los resultados obtenidos en la evaluación de toxicidad oral, dérmica e inhalatoria, ensayos que se realizan en animales o en algunos casos en voluntarios humanos.

La incorporación de nuevas metodologías de testeo permitirá brindar, a la industria local, la tecnología que sustituya el uso de animales de experimentación por procedimientos in vitro e in chemico en la evaluación preclínica de productos agroquímicos, cosméticos y domisanitarios. De esta manera se podrán hacer en nuestro país ensayos que hoy en día se están solicitando al exterior para cumplir con la exigencia de normativas internacionales que excluyen el uso de animales de experimentación.

Esta necesidad fue identificada por la Agencia Nacional de Promoción Científica y Tecnológica, la que habilitó el uso de un subsidio otorgado a la Plataforma Tecnológica EBAL para el equipamiento en el ámbito público del primer laboratorio con capacidad de desarrollar y ofrecer ensayos de toxicidad empleando métodos alternativos validados internacionalmente. Dicho laboratorio, del cual los autores de este trabajo forman parte, funciona en el Instituto de Farmacología de la Facultad de Medicina de la Universidad de Buenos Aires y se ofrece como un ámbito de servicios y de capacitación a otros centros, públicos o privados, interesados en sumarse a la iniciativa de los métodos alternativos. 
Martín Nicolás Rivero, Mariela Lenze, Mercedes Izaguirre, Silvia Wikinski y María Laura Gutiérrez Tendencia al reemplazo de Animales de Experimentación

Nuestro laboratorio es el primero en el país enteramente equipado para realizar ensayos alternativos al uso de animales con fines de investigación toxicológica. Desarrollamos metodologías con alto componente innovador para ponerlas al servicio tanto de los organismos reguladores (ANMAT y SENASA) como de la industria de productos agroquímicos, cosméticos, domisanitarios y/o de las empresas que les prestan servicios para la realización de ensayos preclínicos.

En los últimos años hemos puesto a punto diversas metodologías validadas internacionalmente, entre ellas:

1. Ensayo de citotoxicidad en línea celular (OCDE TG 129) para evaluar toxicidad oral y estimación de la dosis letal 50 (DL50).

2. Ensayos para evaluar daño ocular:

- Ensayo de exposición breve (STE): evalúa la toxicidad producida por una breve exposición a la sustancia de prueba en una línea celular de córnea de conejo (OCDE TG 491).

- Ensayo de opacidad y permeabilidad de córnea bovina (BCOP): determina el potencial corrosivo de una sustancia a partir de un cultivo de córnea bovina (OCDE TG 437). Este método aprovecha el tejido de un animal que ha sido sacrificado con otro fin.

- Ensayo en membrana corioalantoidea de huevo de gallina (HET-CAM): permite evaluar la irritación provocada por una sustancia, usando como modelo huevos de gallina.

3. Comenzamos con la incorporación de las siguientes metodologías para evaluar sensibilización dérmica (reacciones alérgicas) y fototoxicidad:

- Ensayo de reactividad directa de péptidos (DPRA): método in chemico utilizado para predecir si el producto en estudio se une a proteínas de la piel (OCDE TG 442C).

- $\quad$ Test de activación de línea celular humana (h-CLAT) prueba in vitro que predice la activación de células del sistema inmune (OCDE TG 442D).

- Ensayo de fototoxicidad en células 3T3: evalúa el potencial fototóxico de una sustancia mediante un ensayo de toxicidad en cultivo celular (OCDE TG 432).

Las metodologías listadas son de reemplazo total en la mayoría de los casos y de reemplazo parcial en otros, pero la implementación de las mismas lleva a dejar de utilizar animales de laboratorio.

La implementación de estas metodologías podría permitir además ampliar los destinos de exportación de los productos fabricados en Argentina a países que no aceptan información obtenida en animales sin antes haber testeado previamente por un método alternativo. 
Martín Nicolás Rivero, Mariela Lenze, Mercedes Izaguirre, Silvia Wikinski y María Laura Gutiérrez Tendencia al reemplazo de Animales de Experimentación

Reflexiones finales

La producción de sustancias que posiblemente entren en contacto con los seres humanos y con el ambiente no siempre fue evaluada desde el impacto que podrían ocasionar en la salud. Fue necesario que un caso de ceguera llegara a los medios de comunicación para que la sociedad exigiera la evaluación de toxicidad ocular de los productos cosméticos antes que los mismos salgan al mercado. Las metodologías desarrolladas que fueron celebradas en sus inicios, con el paso de los años ocuparon el lugar central entre las críticas debido a la toma de conciencia en cuanto a la crueldad que suponían y a la pobreza en la predicción de los resultados que brindaban.

En respuesta, surgió el Principio de las $3 R$ s, pilar sobre el cual tanto el ámbito público como privado ha ido desarrollando y validando métodos que permiten sustituir el uso de animales de experimentación y aportar resultados confiables sobre el posible riesgo de determinadas sustancias sobre la salud humana. Sin embargo, aún no existe una metodología única que pueda sustituir por completo los ensayos con animales. Existen diversas metodologías disponibles y se continúa trabajando arduamente en perfeccionarlas. La implementación de metodologías alternativas actualmente se basa en plantear una estrategia que utilice una batería de tests in vitro para evaluar toxicidad. Sin embargo no todos los métodos alternativos validados son útiles para todos los productos que se evalúan por lo que es necesario conocer las limitaciones a sus aplicaciones. Hoy en día existen riesgos no cubiertos en torno a la seguridad de nuevos productos que no pueden ser evaluados por las pruebas tradicionales, por lo tanto es necesario que la toxicología profundice sus investigaciones hacia la resolución de esta problemática. Nuevos enfoques están emergiendo bajo el lema "Toxicología del siglo $21 "$ que consiste en el estudio de los mecanismos moleculares que se desencadenan en las células humanas en respuesta a un tóxico. Es posible plantear que el desarrollo de estos nuevos estudios, impulsados por los avances tecnológicos, sea el camino para obtener productos que sean más seguros para el uso humano.

Si bien el reemplazo absoluto de animales de laboratorio por ahora es imposible (en Argentina y en el mundo), particularmente en nuestro país existe un vacío legal sobre la regulación de animales de investigación por lo que es sumamente necesaria la creación de un marco legal. Dicha ley podría dar inicio al debate sobre la incorporación de metodologías alternativas en donde sería pertinente la participación de los entes reguladores, ANMAT y SENASA. 
Martín Nicolás Rivero, Mariela Lenze, Mercedes Izaguirre, Silvia Wikinski y María Laura Gutiérrez Tendencia al reemplazo de Animales de Experimentación

Notas

(1) https://inta.gob.ar/sites/default/files/programa jornada rama 11-10-2017 1.pdf

(2) https://colama2018.com/

Bibliografía

Bal-Price, A., \& Coecke, S. (2011). Guidance on Good Cell Culture Practice (CCP).

Neuromethods, 56, 1-25. https://doi.org/10.1007/978-1-61779-077-5_1

Balls, M. (2015). 19. Russell and Burch after 1959. Alternatives to Laboratory Animals : ATLA, 43(5), P59-60.

Council European Parliament. Directive 2010/63/EU. , (2010).

European Parliament. Registration, Evaluation, Authorisation and Restriction of Chemicals (REACH). , (2013).

European Parliament and of the Council. Directive 86/609/EEC. Protection of animals used for experimental and other scientific purposes. , (1986).

Hartung, T. (2017). Evolution of toxicological science: the need for change. International Journal of Risk Assessment and Management, 20(1/2/3), 21. https://doi.org/10.1504/IJRAM.2017.082570

Kandárová, H., \& Letaáiová, S. (2011). Alternative methods in toxicology: Pre-validated and validated methods. Interdisciplinary Toxicology, 4(3), 107-113. https://doi.org/10.2478/v10102-011-0018-6

LEI N ${ }^{\circ}$ 11.794. Procedimentos para o uso científico de animais. , (2008).

Ley 18.611. UTILIZACIÓN DE ANIMALES EN ACTIVIDADES DE EXPERIMENTACIÓN, DOCENCIA E INVESTIGACIÓN CIENTÍFICA. , (2009).

Mindel, J., \& Wilhelmus, K. R. (2001). THERAPEUTIC REVIEWS. 45(6).

Russell, W. M. S. (1995). The development of the three Rs concept. Alternatives to Laboratory Animals : ATLA, 23(3), 298-304.

Secretaría de Agricultura, Ganadería, P. y A. Resolución 350/99 y su modificatoria Resolución 302/2012 y Anexos. , (2012). 